\title{
Helminth community structure of Scinax nasicus (Anura: Hylidae) from a South American subtropical area
}

\author{
M. I. Hamann*, A. I. Kehr, C. E. González \\ CONICET-CECOAL, ruta 5, km 2.5, W 3400 AMD, Corrientes, Argentina
}

\begin{abstract}
The main goal of this study was to evaluate the relative influence of season, year of study, host body size, and host sex on abundance of helminth species parasitic in the frog Scinax nasicus. A total of 273 frogs was collected between December 2004 and November 2006 over all seasons in Corrientes City, Province of Corrientes, Argentina. Helminth community included 21 taxa, and was dominated particularly by larval trematodes. Infected frogs harboured a maximum of 7 species. Host sex and season played no significant effect in determining infracommunity parasite species abundance. Similarly, species richness was similar for both host sexes, and across time (year and season). However, occurrence of the parasites Opisthogonimus sp. 2 and Travtrema aff. stenocotyle greatly varied over time. Host body size was the main factor for determining infrapopulation structure of Centrorhynchus sp. Species richness was significantly and positively correlated with host body size. Strong associations were observed mainly between metacercariae of some species. The transmission strategies of parasites suggest that this hylid acquires infections by ingestion of infective larvae and through direct contact with larval parasites from aquatic and terrestrial habitats.
\end{abstract}

KEY WORDS: Helminth community $\cdot$ Season $\cdot$ Study period $\cdot$ Host body size $\cdot$ Host sex $\cdot$ Scinax nasicus

\section{INTRODUCTION}

One of the most common ways of characterizing a community is to estimate its diversity, which reflects and combines 2 important aspects, species richness and evenness. Diversity can be measured at the level of either component community or infracommunity; in the latter, the species richness is a subset of the species occurring in the component community (Poulin 1997). In addition, local availability of parasite species and colonization abilities of parasites can affect parasite diversity (reviewed by Esch et. al. 1990), which in turn could affect the degree of interspecific interaction, and distribution of parasite species among infracommunities (see Holmes \& Price 1986). One approach to examine this question would be to study potential species co-occurrence, i.e. testing for positive or negative associations between species present in infracommunities. However, according to Poulin (2005), the interpretation of field study results through a matrix of correlation coefficients must be done cautiously, because when such correlations are found, they do not necessarily imply causation. However, this is one approach that can be taken to understand the functioning of complex natural systems.

Considerations of host sex have often listed as a factor that can influence the number and abundance of parasitic species in vertebrates (Poulin 1996). This approach would be supported by the physiological, behavioural and morphological differences between host sexes. For instance, high testosterone levels can cause immunosuppression in males (Folstad \& Karter 1992), and could operate to create more infection levels than females (Poulin 1996). Females and males also differ with respect to behaviour, e.g. differing reproductive behaviours between sexes can influence exposure to parasite infective stages. The different behaviour of both sexes affects the course of the life cycle 
(Combes 1972), and can also be a strong determinant of differences in parasite occurrences (Gillilland \& Muzzall 1999, Bolek \& Coggins 2001). In addition, sexual dimorphism can also influence parasite intensity or species richness (McAlpine 1997); the larger sex might be more infected because greater host body size provides more surface area for colonization by parasites, i.e. greater probability of skin penetration by cercariae and larval nematodes. Several studies of parasite richness in relation to host body size support this concept (Dogiel 1964, Price 1980, Bell \& Burt 1991, Gregory et al. 1996).

Studies of the influence of biotic factors and external environment on the structure of helminth parasite communities in amphibians have provided important information, showing that anthropogenic perturbations, such as watershed or landscape alterations, can have simultaneous negative and positive effects on distribution, and number of helminth parasite species in infracommunities (Hamann et al. 2006a, McKenzie 2007, King et al. 2008, Marcogliese et al. 2009). Similarly, habitat use (e.g. aquatic, arboreal, terrestrial), relative vagility, feeding preference in terms of foraging behaviour, range of prey species, and host size are key factors determining parasite species richness (Aho 1990, McAlpine 1997, Kehr et al. 2000, Muzzall et al. 2001, Bolek \& Coggins 2003, Hamann et al. 2006b, Yoder \& Coggins 2007).

Additionally, the effect of season on the parasite recruitment period, and the breeding season of the definitive host, have been identified as important factors regulating the structure of amphibian parasite communities (McAlpine 1997, Wetzel \& Esch 1997, Bolek \& Coggins 2001, 2003, Hamann 2004, 2006). The presence of helminths in the final host is also influenced by the effect of seasonal conditions on their intermediate hosts, i.e. changing availability of intermediate hosts and infective stages, as well as parasite transmission ability (Esch et al. 1997, 2002). The timing of larval release is also very important for helminth parasites, because it affects their survival during periods of unfavourable living conditions caused either by climatic factors or by the unavailability of a host (Moravec 1998).

Hamann et al. (2009) reported the helminth taxa of the frog Scinax nasicus, concluding that the adult parasite frequency was very low in the digestive tracts and that larval trematodes were the primary members of this helminth infracommunity; however, this study did not provide any information about the temporal dynamics of the helminth parasite infracommunity and the factors that explain them. In the present study, research was undertaken to test the following hypotheses: (1) helminth infracommunities vary significantly among sampling season and years; (2) large amphibians commonly have more individuals and species of helminths than small amphibians; and (3) helminth populations may serve as markers of diet and habitat use of the frog. These premises were tested by analyzing the composition and structure of parasite communities of $S$. nasicus. This frog is distributed in Paraguay, Northern Argentina, Uruguay, eastern Bolivia, and southwestern Brazil (Frost 2007). In the province of Corrientes, Argentina, S. nasicus is very abundant, and lives sympatrically with $S$. acuminatus; their habitat includes urban and rural areas, and is found mainly on arboreal environments, i.e. on small trees, shrubs and, especially, bromeliads. The S. nasicus diet is dominated by insects (e.g. dipterans, coleopterans), and these frogs principally employ a 'sit and wait' foraging strategy (Duré 1999).

Specifically, the main objectives in this study were to (1) evaluate the magnitude of the influence of season, year of study, host body size, and host sex on the abundance, and number of parasitic helminth species in the frog Scinax nasicus, (2) examine the species affinities (covariation and association) of helminth infracommunities, and (3) analyse the helminth cycles of transmission.

\section{MATERIALS AND METHODS}

Study area. Our study area was located approximately $15 \mathrm{~km}$ east of the city of Corrientes Argentina $\left(27^{\circ} 30^{\prime} \mathrm{S}, 58^{\circ} 45^{\prime} \mathrm{W}\right)$. The 30 ha site is characterized by its wide variety of habitats, containing numerous temporary and semipermanent ponds, and 1 permanent pond, $30 \mathrm{~m}$ long, $15 \mathrm{~m}$ wide and 0.80 meters deep. The vegetation cover is composed of Eichhornia azurea, Nymphaea sp., Hydrocleis nymphoides, Salvinia sp. and Ludwigia peploides; the pond is surrounded by grasslands (Andropogon lateralis). The forest vegetation consists of quebracho Schinopsis balansae, urunday Astronium balansae, and ñandubay Prosopis affinis, with herbaceous strata composed of areas that include gramineous Elyonurus muticus, and terrestrial bromeliads (Aechmea distichantha, Bromelia serra). The mean annual temperature is $23^{\circ} \mathrm{C}$ and the mean annual precipitation is $1500 \mathrm{~mm}$, without a pronounced dry season; however, periods of rain shortages occur every 4 to 6 yr (Carnevali 1994). This area is surrounded by human activities, e.g. weekend houses, sports clubs and pig farming.

Analytical procedure. Adult specimens of Scinax nasicus were collected from terrestrial bromeliads in the study area from December 2004 to August 2005 (Year 1, n = 127), and January 2006 to November 2006 (Year 2, $\mathrm{n}=146$ ). The number of host population samples by year and season of capture and host sex is 
shown in Table 1. Specimens were all hand-captured by 2 people between $18: 00$ and $21: 00 \mathrm{~h}$, using the 'visual encounters survey' sampling technique (Crump $\&$ Scott 1994). Frogs were transported live to the laboratory, killed in a chloroform $\left(\mathrm{CHCl}_{3}\right)$ solution, and dissected following standard protocols (Goater \& Goater 2001). Their snout-vent length (SVL) and body weight were recorded. At necropsy, hosts were sexed and the oesophagus, stomach, gut, lungs, liver, urinary bladder, kidneys, body cavity, musculature, skin, and brain examined for parasites. Helminths were observed in vivo, counted, killed in hot distilled water and preserved in $70 \%$ ethyl alcohol. Digeneans, cestodes, and acanthocephalans were stained with hydrochloric carmine, cleared in creosote, and mounted in Canada balsam. Nematodes were cleared in glycerine or lactophenol, and examined as temporary mounts. Helminths were identified using Yamaguti (1961, 1963, 1975), Jones (1987), Anderson (2000), Gibson et al. (2002), and Jones et al. (2005). Specimens of various parasite species were deposited in the Helminthological Collection of the Centro de Ecología Aplicada del Litoral (CECOAL-CONICET), Corrientes City, Corrientes, Argentina (see Table 2).

Statistical analysis. Infection prevalence, intensity and abundance were calculated for helminths according to Bush et al. (1997). The measures of community richness and diversity employed included the total number of helminth species (= richness), Shannon in$\operatorname{dex}\left(H^{\prime}\right)$ (Shannon \& Weaver 1949), and evenness $\left(J^{\prime}\right)$ as $H^{\prime} / H_{\text {max. }}^{\prime}$ (Pielou 1966, Zar 1996). Mean helminth species richness is the sum of helminth species, per individual frog, divided by the total sample size. A distribution

Table 1. Scinax nasicus. Number of sampled populations by year of study, season of capture, and host sex. Season dates are summer (21 Dec-20 Mar), autumn (21 Mar-20 Jun), winter (21 Jun-20 Sep) and spring (21 Sep-20 Dec). Chi-square $\left(\chi^{2}\right)$ values in Table are for comparisons between numbers of each sex collected within seasons. For the comparison of total frogs collected in each year, $\chi^{2}=1.33$. All $\chi^{2}$ values are non-significant ( $p>0.05)$

\begin{tabular}{|lcccc|}
\hline Season & $\begin{array}{c}\text { Total } \\
\text { frogs }\end{array}$ & $\begin{array}{c}\text { No. of } \\
\text { male }\end{array}$ & $\begin{array}{c}\text { No. of } \\
\text { female }\end{array}$ & $\chi^{2}$ \\
\hline Year 1 (2004-2005, & $\mathbf{n}=\mathbf{1 2 7})$ & & & \\
Summer & 53 & 26 & 27 & 0.04 \\
Autumn & 39 & 21 & 18 & 0.26 \\
Winter & 35 & 23 & 12 & 3.49 \\
Year 2 (2006, n = 146) & & & & \\
Summer & 20 & 10 & 10 & 0.05 \\
Autumn & 37 & 14 & 23 & 2.22 \\
Winter & 53 & 23 & 30 & 0.94 \\
Spring & 36 & 16 & 20 & 0.47 \\
Total & 273 & 133 & 140 & 0.18 \\
\hline
\end{tabular}

of infracommunity richness values was tested to see if they indicated some sort of structure between the number of helminth species vs. number of frogs. The diversity index was used with decimal logarithms $\left(\log _{10}\right)$. Berger-Parker index of dominance $(d)$ was used to determine the most abundant species (Magurran 2004).

Helminth communities were classified at the infracommunity (all helminth infrapopulations within a single Scinax nasicus) and component community (all helminth infracommunities within a population of $S$. nasicus) levels. Chi-square $\left(\chi^{2}\right)$ test was used for comparing the sex ratio of the frogs. Mann-Whitney's $U$ test was used to test for differences in helminth richness among the sex. Comparison of 2 proportions (Z-test) was used to test for differences in prevalence of helminth infection between sexes. Comparison of $k$ proportion $\left(\chi^{2}\right)$ was used to test for differences in prevalence between seasons. Kruskal-Wallis test $(H)$ was used to test for differences in species richness between season and year. Pearson's coefficient correlation (r) was used to indicate the relationship between host body size, parasitic abundance, and infracommunity descriptors. Spearman's Rank test $\left(\mathrm{r}_{\mathrm{S}}\right)$ was used to calculate possible species co-variation. The species associations was analysed with a $\chi^{2}$ test, with Yates correlation. The degree of aggregation of different species of parasites was calculated by the index of dispersion, which indicated the departure of the distribution from randomness (Elliot 1971, Southwood 1978):

$$
\chi^{2}=\frac{s^{2}(\mathrm{~N}-1)}{\bar{X}}
$$

where $s^{2}=$ variance, $\mathrm{N}=$ number of samples and $\bar{x}=$ mean. This index of dispersion will often depart from unity, and the significance of these departures is assessed by reference to a table of $\chi^{2}$ with $n-1$ degrees of freedom.

The abundance of infection of each parasite species and its relation with biotic and abiotic factors was analysed thought a multivariate analyses of variance (MANOVA), previously normalization of the data by $\log _{10}(x+1)$ transformation. When MANOVA Wilks' Lambda was significant, a univariate test (ANOVA) was realized for each dependent variable; the probabilities were calculated according to the 'sequential Bonferroni' procedure (Holm 1979), because it provides great control over Type I errors. The spring data was not considered in MANOVA, because sampling in this season was only done in one year. For abundance vs. host body size, co-variation and associations of helminth species, and the effect of interacting factors, the species considered were those that had at least $10 \%$ occurrences in each of the amphibians' populations (6 species). The software used were Xlstat 7.5 (Addinsoft 2004) and SYSTAT 7. 


\section{RESULTS}

\section{Community structure analysis}

The helminth component community for this frog population consisted of 21 species of helminths (Table 2). The predominant groups of parasites were the trematodes $(71 \%)$ followed by the nematodes $(19 \%)$; the other groups of parasites were represented by only 1 species (Cestoda: Cylindrotaenia sp.; Acanthocephala: Centrorhynchus sp.). Helminth diversity and evenness were 0.54 and 0.41 , respectively. Bursotrema tetracotyloides was the dominant species $(d=$ $0.59)$. The majority $(76 \%)$ of the helminth parasite spe- cies of Scinax nasicus showed a significant $(p<0.025)$ aggregated pattern of distribution. Parasites were found in kidneys, muscle, mesenteries, body cavity and pharyngeal zone at a high level of infection (Table 2). Of all the helminth species for which adults were examined, 4 are assumed to have indirect life cycles (Glypthelmins sp., Catadiscus inopinatus, Gorgoderina rochalimai and Mesocoelium monas), and 3 are assumed to have direct life cycles (Cylindrotaenia sp., Cosmocerca podicipinus and Cosmocerca parva) (Table 3).

At the infracommunity level, the mean helminth richness was $2.07 \pm 1.26$ (maximum $=7$ ) species per infected frog. The observed distribution of helminth in-

Table 2. Helminths infecting Scinax nasicus. Accession numbers of submitted helminth samples (Helminthological Collection, Centro de Ecología Aplicada del Litoral [CECOAL], Corrientes, Argentina), site of infection (Li: large intestine; Si: small intestine; UB: urinary bladder; K: Kidneys; Mu: muscle; Me: Mesenteries; Bc: Body cavity; Pz: Pharyngeal zone; L: liver; Ss: Serous of stomach), prevalence $(\%)$, mean $( \pm \mathrm{SD})$ abundance, mean $( \pm \mathrm{SD})$ intensity, and index of dispersion $(I)$. $I$-values in bold are aggregated distribution $(p<0.025)$ and normal types are random distribution $(0.025 \leq p \leq 0.975)$; degrees of freedom $=272$ in all cases. NA: not available (specimen not submitted); -: mean intensity not calculated, because only 1 host was infected

\begin{tabular}{|c|c|c|c|c|c|c|c|}
\hline Helminth species & Stage & $\begin{array}{l}\text { CECOAL } \\
\text { access. no. }\end{array}$ & $\begin{array}{l}\text { Site of } \\
\text { infection }\end{array}$ & $\begin{array}{c}\text { Prevalence } \\
(\%)\end{array}$ & $\begin{array}{c}\text { Mean } \\
\text { abundance }\end{array}$ & $\begin{array}{l}\text { Mean } \\
\text { intensity }\end{array}$ & $I$ \\
\hline \multicolumn{8}{|l|}{ Trematoda } \\
\hline $\begin{array}{l}\text { Catadiscus inopinatus } \\
\text { Freitas, } 1841\end{array}$ & Adult & 05012412 & $\mathrm{Li}$ & 11.0 & $0.15 \pm 047$ & $1.33 \pm 0.65$ & 407.07 \\
\hline Glypthelmins sp. & Adult & 05012416 & $\mathrm{Si}$ & 0.4 & $0.01 \pm 0.18$ & - & 816.00 \\
\hline $\begin{array}{l}\text { Gorgoderina rochalimai } \\
\text { Pereira and Cuocolo, } 1940\end{array}$ & Adult & 06093013 & Ub & 0.4 & $0.004 \pm 0.06$ & - & 272.00 \\
\hline $\begin{array}{l}\text { Mesocoelium monas } \\
\text { (Rudolphi, 1819) }\end{array}$ & Adult & 06083107 & $\mathrm{Si}$ & 0.4 & $0.007 \pm 0.12$ & - & 571.20 \\
\hline $\begin{array}{l}\text { Bursotrema tetracotyloides } \\
\text { Szidat, } 1960\end{array}$ & Larva & 05032809 & $\mathrm{~K}$ & 42.1 & $17.20 \pm 52.39$ & $40.83 \pm 74.50$ & 43411.20 \\
\hline $\begin{array}{l}\text { Lophosyciadiplostomum } \\
\text { aff. nephrocystis }\end{array}$ & Larva & 04121324 & K & 0.4 & $0.01 \pm 0.24$ & - & 1060.08 \\
\hline $\begin{array}{l}\text { Travtrema aff. stenocotyle } \\
\text { Cohn, } 1902\end{array}$ & Larva & 06093005 & $\mathrm{Mu} \mathrm{Me} \mathrm{Bc} \mathrm{Pz}$ & 28.9 & $1.70 \pm 7.15$ & $5.86 \pm 12.34$ & 8214.40 \\
\hline Styphlodora sp. & Larva & 06083127 & K Mu L & 4.0 & $0.15 \pm 1.17$ & $3.73 \pm 4.51$ & 2475.20 \\
\hline Opisthogonimus sp. 1 & Larva & 06073113 & $\mathrm{Me} \mathrm{Bc}$ & 2.9 & $0.07 \pm 0.48$ & $2.38 \pm 1.58$ & 897.60 \\
\hline Opisthogonimus sp. 2 & Larva & 06093008 & $\mathrm{Mu} \mathrm{Me} \mathrm{Bc} \mathrm{Pz}$ & 62.3 & $6.69 \pm 11.93$ & $10.74 \pm 13.61$ & 5793.60 \\
\hline Nephrostomum sp. & Larva & 05042119 & $\mathrm{~L}$ & 1.1 & $0.03 \pm 0.28$ & $2.33 \pm 1.25$ & $\mathbf{7 8 8 . 8 0}$ \\
\hline $\begin{array}{l}\text { Unknown echinostomatid } \\
\text { species } 1\end{array}$ & Larva & NA & $\mathrm{Pz}$ & 16.8 & $2.34 \pm 10.89$ & $13.89 \pm 23.30$ & 13763.20 \\
\hline $\begin{array}{l}\text { Unknown echinostomatid } \\
\text { species } 2\end{array}$ & Larva & NA & K & 0.4 & $0.01 \pm 0.24$ & - & 1060.80 \\
\hline Unknown plagiorchid species & Larva & 05042118 & $\mathrm{Mu} \mathrm{Bc}$ & 1.1 & $0.08 \pm 0.87$ & $7.33 \pm 4.03$ & 2556.80 \\
\hline Unknown strigeid species & Larva & 05062909 & $\mathrm{BC}$ & 2.2 & $0.03 \pm 0.18$ & $1.17 \pm 0.37$ & 326.40 \\
\hline \multicolumn{8}{|l|}{ Nematoda } \\
\hline $\begin{array}{l}\text { Cosmocerca podicipinus } \\
\text { Baker and Vaucher, } 1984\end{array}$ & Adult & 05072802 & $\mathrm{Li}$ & 1.5 & $0.01 \pm 0.12$ & $1.00 \pm 0.00$ & 252.96 \\
\hline $\begin{array}{l}\text { Cosmocerca parva } \\
\text { Travassos, } 1925\end{array}$ & Adult & 04121316 & $\mathrm{Li}$ & 0.4 & $0.004 \pm 0.06$ & - & 272.00 \\
\hline Physaloptera sp. & Larva & 06073104 & Ss & 0.4 & $0.004 \pm 0.06$ & - & 272.00 \\
\hline $\begin{array}{l}\text { Unknown rhabdochonid } \\
\text { species }\end{array}$ & Larva & 05042115 & Ss & 1.1 & $0.01 \pm 0.10$ & $1.00 \pm 0.00$ & 272.00 \\
\hline \multicolumn{8}{|l|}{ Acanthocephala } \\
\hline Centrorhynchus sp. & Larva & 06083118 & Ss Me & 27.1 & $0.69 \pm 1.50$ & $2.53 \pm 1.92$ & 897.60 \\
\hline Cylindrotaenia sp. & Adult & 06083118 & $\mathrm{Si}$ & 1.8 & $0.07 \pm 0.64$ & $4.00 \pm 2.53$ & 1496.00 \\
\hline
\end{tabular}


Table 3. Summary of helminth taxa, life cycle, transmission of larval stages and definitive host (DH) of parasites in Scinax nasicus from Corrientes, Argentina. Sources are for life cycles of congener species or species of the same family

\begin{tabular}{|c|c|c|c|c|}
\hline Helminth & Life cycle & Transmission & DH & Source \\
\hline $\begin{array}{l}\text { Catadiscus } \\
\text { inopinatus }\end{array}$ & Indirect & $\begin{array}{l}\text { Cercariae encysted on substrates (e.g. grass and aquatic } \\
\text { vegetation) or on skin of tadpoles; metacercariae ingested } \\
\text { with vegetation by definitive host, e.g. Catadiscus uruguayensis } \\
\text { Freitas and Lent, } 1939\end{array}$ & Amphibian & Ostrowski de Núñez (1979a) \\
\hline Glypthelmins sp. & Indirect & $\begin{array}{l}\text { Skin penetration by cercariae in tadpoles and adult frogs. Oral } \\
\text { ingestion by metacercariae encysted in sloughed skin of } \\
\text { tadpoles by definitive host, e.g. G. quieta Stafford, 1900) }\end{array}$ & Amphibian & $\begin{array}{l}\text { Leigh (1946), } \\
\text { Smyth \& Smyth (1980) }\end{array}$ \\
\hline $\begin{array}{l}\text { Gorgoderina } \\
\text { rochalimai }\end{array}$ & Indirect & $\begin{array}{l}\text { Oral ingestion of cercariae by tadpoles or aquatic insect larvae; } \\
\text { metacercariae encysted in body cavity ingested by definitive } \\
\text { host, e.g. G. attenuata Stafford, } 1902\end{array}$ & Amphibian & Bolek et al. (2009) \\
\hline $\begin{array}{l}\text { Mesocoelium } \\
\text { monas }\end{array}$ & Indirect & $\begin{array}{l}\text { Cercariae encysted in viscera of intermediate snail, or emerge } \\
\text { and encyst on any object they contact, including vegetation, } \\
\text { e.g. M. monodi Dollfus, } 1929\end{array}$ & Amphibian & Thomas (1965) \\
\hline $\begin{array}{l}\text { Bursotrema } \\
\text { tetracotyloides }\end{array}$ & Indirect & $\begin{array}{l}\text { Presumably skin penetration by cercariae in amphibians, e.g. } \\
\text { Fibricola texensis Chandler, 1947; metacercariae encapsulated } \\
\text { in kidneys ingested by definitive host }\end{array}$ & Mammal & Yamaguti (1975) \\
\hline $\begin{array}{l}\text { Lophosyciadiplo- } \\
\text { stomum aff. } \\
\text { nephrocystis }\end{array}$ & Indirect & $\begin{array}{l}\text { Skin penetration by cercariae in tadpoles, e.g. Neodiplostomum } \\
\text { buteonis Dubois and Rausch, 1948; metacercariae encapsulated } \\
\text { in kidney ingested by definitive host }\end{array}$ & Bird & Pearson (1960) \\
\hline $\begin{array}{l}\text { Travtrema aff. } \\
\text { stenocotyle }\end{array}$ & Indirect & $\begin{array}{l}\text { Skin penetration by cercariae in tadpoles; metacercariae } \\
\text { encysted in muscle, mesentery, body cavity and pharyngeal zone } \\
\text { ingested by definitive host, e.g. T. stenocotyle Cohn, } 1902\end{array}$ & Snake & Ostrowski de Núñez (1979b) \\
\hline Styphlodora sp. & Indirect & $\begin{array}{l}\text { Skin penetration by cercariae in tadpoles, e.g. Metaleptophallus } \\
\text { gracillimus (Lühe, 1909); metacercariae encysted in kidney, } \\
\text { muscle and liver ingested by definitive host }\end{array}$ & Snake & Grabda-Kazubska (1963) \\
\hline $\begin{array}{l}\text { Opisthogonimus } \\
\text { sp. } 1\end{array}$ & Indirect & $\begin{array}{l}\text { Skin penetration by cercariae in tadpoles, e.g. Metaleptophallus } \\
\text { gracillimus (Lühe, 1909); metacercariae encysted in mesentery } \\
\text { and body cavity ingested by definitive host }\end{array}$ & Snake & Grabda-Kazubska (1963) \\
\hline $\begin{array}{l}\text { Opisthogonimus } \\
\text { sp. } 2\end{array}$ & Indirect & $\begin{array}{l}\text { Skin penetration by cercariae in tadpoles, e.g. Metaleptophallus } \\
\text { gracillimus (Lühe, 1909); metacercariae encysted in muscle, } \\
\text { mesentery, body cavity and pharyngeal zone ingested by } \\
\text { definitive host }\end{array}$ & Snake & Grabda-Kazubska (1963) \\
\hline Nephrostomum sp. & Indirect & $\begin{array}{l}\text { Natural orifices, e.g. nostrils, mouth, penetrating by cercariae } \\
\text { e.g. Episthmium suspensum (Braun, 1901); metacercariae } \\
\text { encysted in liver ingested by definitive host }\end{array}$ & Bird & Ostrowski de Núñez (1974) \\
\hline $\begin{array}{l}\text { Unknown echino- } \\
\text { stomatid species } 1\end{array}$ & Indirect & $\begin{array}{l}\text { Natural orifices, e.g. nostrils, mouth, penetrating by cercariae in } \\
\text { tadpoles, e.g. Episthmium suspensum (Braun, 1901); } \\
\text { metacercariae encysted in pharyngeal zone ingested by } \\
\text { definitive host }\end{array}$ & $\begin{array}{l}\text { Reptile/Bird/ } \\
\text { Mammal }^{\mathrm{a}}\end{array}$ & Ostrowski de Núñez (1974) \\
\hline $\begin{array}{l}\text { Unknown echino- } \\
\text { stomatid species } 2\end{array}$ & Indirect & $\begin{array}{l}\text { Natural orifices, e.g. nostrils, mouth, penetrating by cercariae in } \\
\text { tadpoles, e.g. Episthmium suspensum (Braun, 1901); } \\
\text { metacercariae encysted in kidney ingested by definitive host }\end{array}$ & $\begin{array}{l}\text { Reptile/Bird/ } \\
\text { Mammal }^{\mathrm{a}}\end{array}$ & Ostrowski de Núñez (1974) \\
\hline $\begin{array}{l}\text { Unknown } \\
\text { plagiorchid species }\end{array}$ & Indirect & $\begin{array}{l}\text { Skin penetration of cercariae in tadpoles, e.g. Metaleptophallus } \\
\text { gracillimus (Lühe, 1909); metacercariae encysted in muscle } \\
\text { and body cavity ingested by definitive host }\end{array}$ & $\begin{array}{l}\text { Reptile/Bird/ } \\
\text { Mammal }^{\mathrm{a}}\end{array}$ & Grabda-Kazubska (1963) \\
\hline $\begin{array}{l}\text { Unknown } \\
\text { strigeid species }\end{array}$ & Indirect & $\begin{array}{l}\text { Natural orifices, e.g. branchial, mouth, penetrating by cercariae } \\
\text { in tadpoles, e.g. Apharyngostrigea pipientis (Oliver 1940); meta- } \\
\text { cercariae encysted in body cavity ingested by definitive host }\end{array}$ & $\begin{array}{c}\text { Bird/ } \\
\text { Mammal }^{\mathrm{a}}\end{array}$ & Yamaguti (1975) \\
\hline $\begin{array}{l}\text { Cosmocerca } \\
\text { podicipinus }\end{array}$ & Direct & $\begin{array}{l}\text { Skin penetration by infective larvae in amphibians, e.g. } \\
\text { C. commutata (Diesing, 1851) }\end{array}$ & Amphibian & Fotedar \& Tikoo (1968) \\
\hline Cosmocerca parva & Direct & $\begin{array}{l}\text { Skin penetration by infective larvae in amphibians, e.g. } \\
\text { C. commutata (Diesing, 1851) }\end{array}$ & Amphibian & Fotedar \& Tikoo (1968) \\
\hline Physaloptera sp. & Indirect & $\begin{array}{l}\text { Oral ingestion of eggs by insects. Larvae }\left(\mathrm{L}_{3}\right) \text { encapsulated in } \\
\text { intestine ingested by paratenic, e.g. amphibian, and defini- } \\
\text { tive hosts, e.g. P. maxillaris Molin, } 1860\end{array}$ & $\begin{array}{l}\text { Reptile/Bird/ } \\
\text { Mammal }^{\mathrm{a}}\end{array}$ & $\begin{array}{l}\text { Cawthorn \& Anderson (1976), } \\
\text { Anderson (2000) }\end{array}$ \\
\hline $\begin{array}{l}\text { Unknown rhabdo- } \\
\text { chonid species }\end{array}$ & Indirect & $\begin{array}{l}\text { Oral ingestion of eggs by aquatic insects and amphipods. } \\
\text { Larvae }\left(\mathrm{L}_{3}\right) \text { encapsulated in body cavity ingested by defini- } \\
\text { tive hosts, e.g. Rhabdochona phoxini Moravec, } 1968\end{array}$ & $\begin{array}{l}\text { Fish/ } \\
\text { Amphibian }^{\mathrm{a}}\end{array}$ & $\begin{array}{l}\text { Moravec (1977), } \\
\text { Moravec \& Kaiser (1994) }\end{array}$ \\
\hline Centrorhynchus sp. & Indirect & $\begin{array}{l}\text { Oral ingestion of eggs by insects and probably terrestrial isopods. } \\
\text { Larvae }\left(\mathrm{L}_{3}\right) \text { encapsulated in body cavity ingested by paratenic host } \\
\text { e.g. amphibian, and definitive host, e.g. C. aluconis Golvan, } 1960\end{array}$ & Bird & Schmidt (1985) \\
\hline Cylindrotaenia sp. & Direct & $\begin{array}{l}\text { Probably, oral ingestion of embryonated eggs by definitive host, } \\
\text { e.g. C. americana Jewell, } 1916\end{array}$ & Amphibian & Prudhoe \& Bray (1982) \\
\hline
\end{tabular}


fracommunity species richness showed a good fit to Poisson distribution $\left(\chi^{2}=10.78\right.$, df $\left.=6, \mathrm{p}=0.095\right)$ (Fig. 1). Mean values of helminth diversity and evenness were $0.22 \pm 0.17$ and $0.52 \pm 0.37$, respectively. Two correlations between helminth species occurrence were significant: one was negative $(C$. inopinatus vs. Bursotrema tetracotyloides), and one was positive (unknown echinostomatid species 1 vs. B. tetracotyloides) (Table 4). Three associations were found among the 6 helminth species considered: Opisthogonimus sp. $2+B$. tetracotyloides $\left(\chi^{2}=8.14\right.$, df $=1, \mathrm{p}<$ 0.05), unknown echinostomatid species $1+B$. tetracotyloides $\left(\chi^{2}=18.05, \mathrm{df}=1, \mathrm{p}<0.05\right)$ and Opisthogonimus sp. $2+$ Travtrema aff. stenocotyle $\left(\chi^{2}=6.30\right.$, $\mathrm{df}=1, \mathrm{p}<0.05)$.

\section{Infection in relation to host sex and body size}

Of the 273 frogs examined, the infection prevalence was $89 \%$; there was no significant difference in the prevalence of infected females (52\%) vs. males $(48 \%)$ $(Z=0.62, \mathrm{df}=1, \mathrm{p}>0.05)$. Parasite richness did not

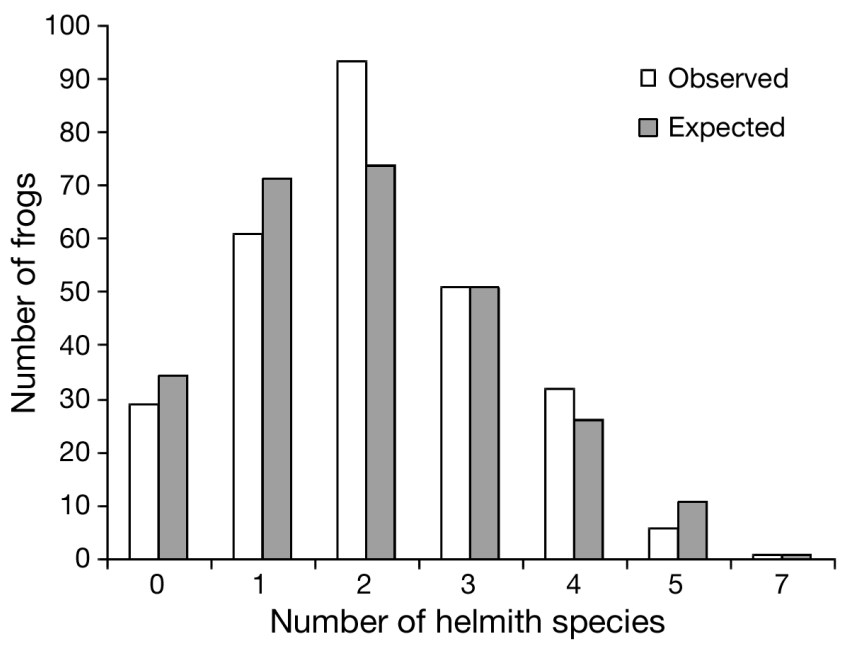

Fig. 1. Observed and expected frequency distribution of helminth infracommunity species richness in Scinax nasicus, according to the Poisson distribution pattern show any relationship with host sex (Mann-Whitney $U$-test $\left.=8212.50, \mathrm{p}>0.05, \mathrm{n}_{\text {female }}=126, \mathrm{n}_{\text {male }}=118\right)$.

Total length of the frogs ranged from 12.50 to 37.50 $(24.47 \pm 5.18) \mathrm{mm}$, and weight ranged from 0.15 to 3.78 $(0.95 \pm 0.60) \mathrm{g}$. Infracommunity descriptors and abundance of helminth species were primarily not significantly correlated with body size of the host (Table 5). Nevertheless, significant correlation were observed for richness vs. length $(\mathrm{r}=0.149, \mathrm{n}=244, \mathrm{p}<0.05)$ and abundance of Centrorhynchus sp. vs. host body size (length: $\mathrm{r}=0.226, \mathrm{n}=239, \mathrm{p}<0.05$; weight: $\mathrm{r}=0.231$, $\mathrm{n}=239$ ).

Body sizes of males and female frogs did not show significant difference (length: Mann-Whitney $U$-test $=$ 9918.50, $\mathrm{p}>0.05, \mathrm{n}_{\text {female }}=140, \mathrm{n}_{\text {male }}=133$; weight: Mann-Whitney $U$-test $=9873.00, \mathrm{p}>0.05, \mathrm{n}_{\text {female }}=$ $140, \mathrm{n}_{\text {male }}=133$ ). The length of female frogs ranged from 15.00 to $37.50(25.09 \pm 4.94) \mathrm{mm}$, and weight ranged from 0.10 to $3.78(1.03 \pm 0.65) \mathrm{g}$. These variables were not significantly correlated with most of the helminth parasites and infracommunity descriptors (Table 5). The length of male frogs ranged from 12.50 to $36.00(24.29 \pm 5.06) \mathrm{mm}$, and weight ranged from 0.15 to $2.47(0.92 \pm 0.54) \mathrm{g}$. These variables were significantly correlated with infracommunity descriptors, and with the abundance of 2 larval stages of helminths (Table 5).

\section{Influence of year, season and sex on abundance and richness of parasites}

MANOVA was performed using data from the year, season and sex as classification factors, while the dependent variable were the abundance of following 6 species: Centrorhynchus sp., Catadiscus inopinatus, Bursotrema tetracotyloides, echinostomatid species 1, Opisthogonimus sp. 2, and Travtrema aff. stenocotyle. These results showed that season (MANOVA Wilks' Lambda $\left.=0.94, F_{12,378}=0.96, p=0.49\right)$, and sex (MANOVA Wilks' Lambda $=0.98, F_{6,189}=0.67, \mathrm{p}=$ $0.67)$ played no significant effect in determining infracommunity species abundance; nevertheless, sig-

Table 4. Covariation based on Spearman Rank correlations $\left(\mathrm{r}_{\mathrm{S}}\right)$, among 6 helminth species common in Scinax nasicus from Corrientes, Argentina. Significant values $(\mathrm{p}<0.05)$ in bold

\begin{tabular}{|c|c|c|c|c|c|c|c|}
\hline Species & Table ID & (1) & $(2)$ & (3) & (4) & (5) & (6) \\
\hline Catadiscus inopinatus & $(1)$ & 1.000 & & & & & \\
\hline Unknown echinostomatid species 1 & (2) & 0.101 & 1.000 & & & & \\
\hline Opisthogonimus sp. 2 & (3) & 0.001 & 0.098 & 1.000 & & & \\
\hline Travtrema aff. stenocotyle & (4) & -0.037 & 0.105 & -0.127 & 1.000 & & \\
\hline Bursotrema tetracotyloides & (5) & -0.159 & 0.381 & -0.091 & -0.029 & 1.000 & \\
\hline Centrorhynchus sp. & (6) & -0.087 & -0.008 & 0.028 & -0.041 & -0.094 & 1.000 \\
\hline
\end{tabular}


Table 5. Pearson correlation (r) between helminth parasites and sex size of Scinax nasicus. Significant values ( $\mathrm{p}<0.05)$ in bold

\begin{tabular}{|c|c|c|c|c|c|c|}
\hline \multirow[t]{2}{*}{ Helminth species } & \multicolumn{2}{|c|}{ _ Total frogs } & \multicolumn{2}{|c|}{$\ldots$ Male } & \multicolumn{2}{|c|}{ Female } \\
\hline & Length & Weight & Length & Weight & Length & Weight \\
\hline \multicolumn{7}{|l|}{ Parasitic abundance } \\
\hline Catadiscus inopinatus & -0.037 & -0.071 & -0.025 & -0.045 & -0.057 & -0.097 \\
\hline Unknown echinostomatid species 1 & -0.076 & -0.077 & 0.039 & 0.042 & -0.174 & -0.170 \\
\hline Opisthogonimus sp. 2 & -0.060 & -0.060 & -0.042 & -0.060 & -0.044 & -0.038 \\
\hline Travtrema aff. stenocotyle & 0.051 & 0.018 & 0.022 & -0.034 & 0.094 & 0.073 \\
\hline Bursotrema tetracotyloides & 0.041 & 0.081 & 0.150 & 0.185 & -0.034 & 0.006 \\
\hline Centrorhynchus sp. & 0.226 & 0.231 & 0.190 & 0.191 & 0.270 & 0.270 \\
\hline \multicolumn{7}{|l|}{ Community descriptors } \\
\hline Diversity & 0.123 & 0.086 & 0.228 & 0.177 & 0.018 & 0.002 \\
\hline Evenness & 0.118 & 0.081 & 0.247 & 0.184 & -0.028 & -0.029 \\
\hline Richness & 0.149 & 0.122 & 0.264 & 0.220 & 0.038 & 0.039 \\
\hline
\end{tabular}

nificant differences in the presence of helminth species were found between years (MANOVA Wilks' Lambda $\left.=0.84, F_{6,189}=5.82, p=0.0001\right)$. In the last case, an a posteriori univariate test (ANOVA) was calculated (using probabilities according to 'sequential Bonferroni' criteria). These data showed significant differences between years in the parasite abundance
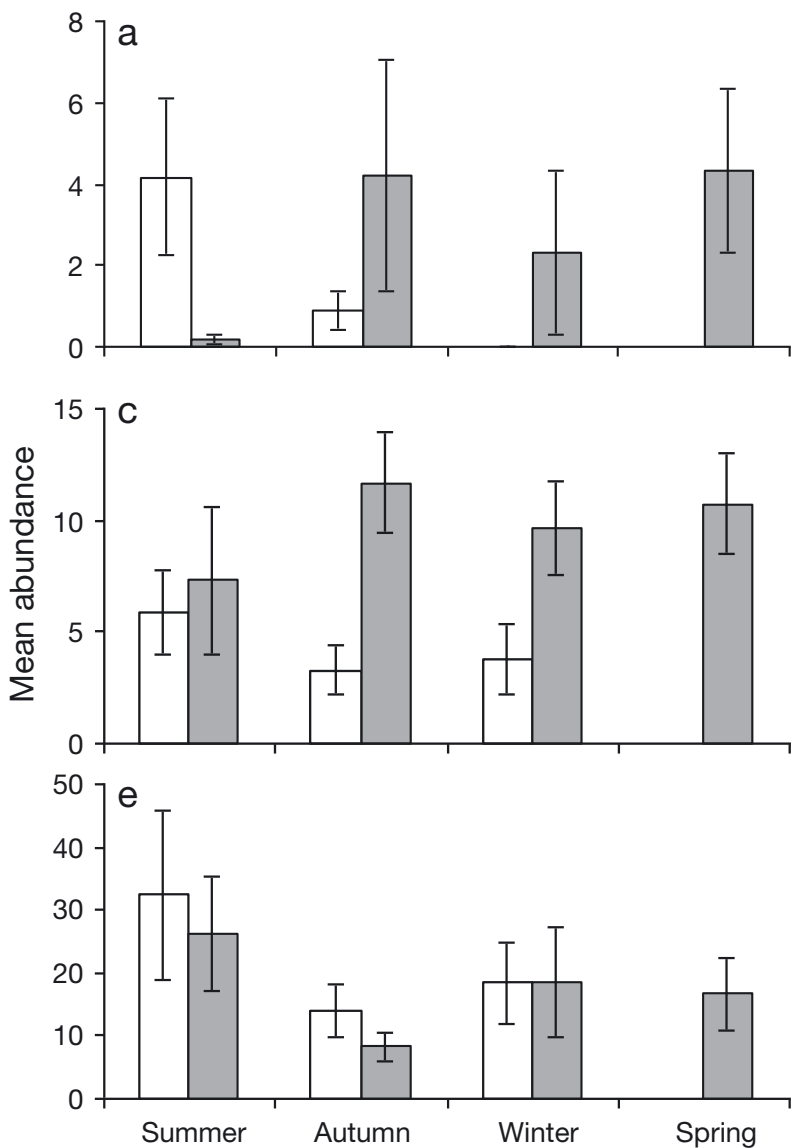

of Opisthogonimus sp. $2(F=23.86, \mathrm{df}=1, \mathrm{p}=0.001)$, and $T$. aff. stenocotyle $(F=6.70, \mathrm{df}=1, \mathrm{p}=0.010)$. Similar results were observed for mean abundance of both species (Fig. 2c,d). All factor interactions were non-significant ( $p>0.05)$.

Results of $k$ proportion comparison in helminth prevalence infections showed that season had a signif-
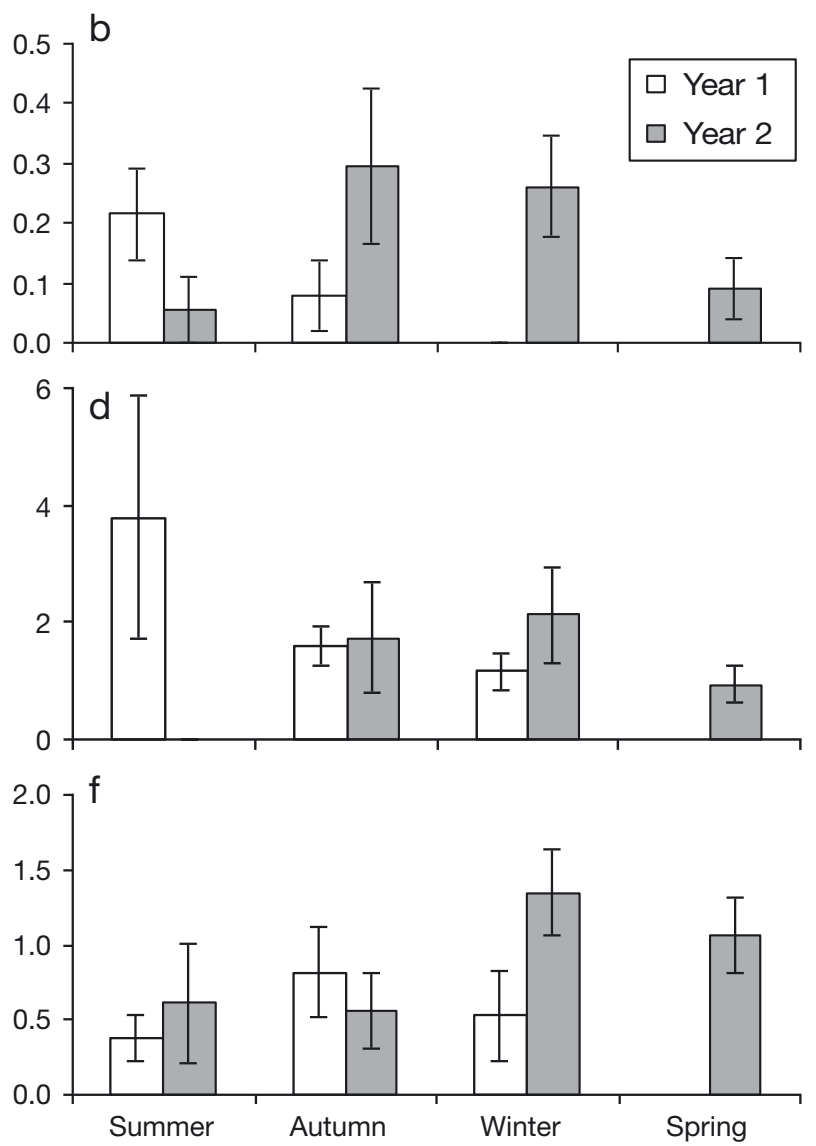

Fig. 2. Seasonal variation in mean $( \pm \mathrm{SE})$ helminth parasite abundance in Scinax nasicus. (a) Unknown echinostomatid species 1, (b) Catadiscus inopinatus, (c) Opisthogonimus sp. 2, (d) Travtrema aff. stenocotyle, (e) Bursotrema tetracotyloides, (f) Centrorhynchus sp. 
Table 6. Results of $k$ proportions comparison in helminth prevalence infections among the seasons and years. Significant values $(\mathrm{p}<0.05)$ in bold

\begin{tabular}{|lccccccc|}
\hline Helminth species & \multicolumn{3}{c}{ Year 1} & \multicolumn{4}{c|}{ Year 2 } \\
& $\chi^{2}$ & $\mathrm{df}$ & $\mathrm{p}$ & $\chi^{2}$ & $\mathrm{df}$ & $\mathrm{p}$ \\
\hline Catadiscus inopinatus & 5.49 & 2 & 0.064 & 3.79 & 3 & 0.285 \\
Unknown echinostomatid species 1 & 4.38 & 2 & 0.112 & 5.75 & 3 & 0.125 \\
Opisthogonimus sp. 2 & 4.48 & 2 & 0.106 & 2.45 & 3 & 0.484 \\
Travtrema aff. stenocotyle & 1.14 & 2 & 0.566 & 9.10 & 3 & $\mathbf{0 . 0 2 8}$ \\
Bursotrema tetracotyloides & 2.58 & 2 & 0.275 & 7.38 & 3 & 0.061 \\
Centrorhynchus sp. & 1.35 & 2 & 0.510 & 12.30 & 3 & $\mathbf{0 . 0 0 6}$ \\
\hline
\end{tabular}

\section{DISCUSSION}

The analysis presented here shows that Scinax nasicus hosts a high richness (21 species) of larval and adult helminths, with a maximum of 7 species per frog, with Bursotrema tetracotyloides as the dominant species. Only 1 species (Opisthogonimus sp. 2) shows a high prevalence $(>60 \%)$ of infection, for which $S$. nasicus represents a specific intermediate host; this icant effect only on Travtrema aff. stenocotyle and Centrorhynchus sp. in the second year (Table 6, Fig. 3d,f).

Infracommunity richness was not related to years (Mann-Whitney $U$-test $=6713.00, \mathrm{p}>0.05, \mathrm{n}_{\text {Year } 1}=$ $\left.110, \mathrm{n}_{\text {Year } 2}=134\right)$, or seasons (Kruskal-Wallis $H$-test $=$ 6.59, $\mathrm{p}>0.05, \mathrm{df}=3$ ).
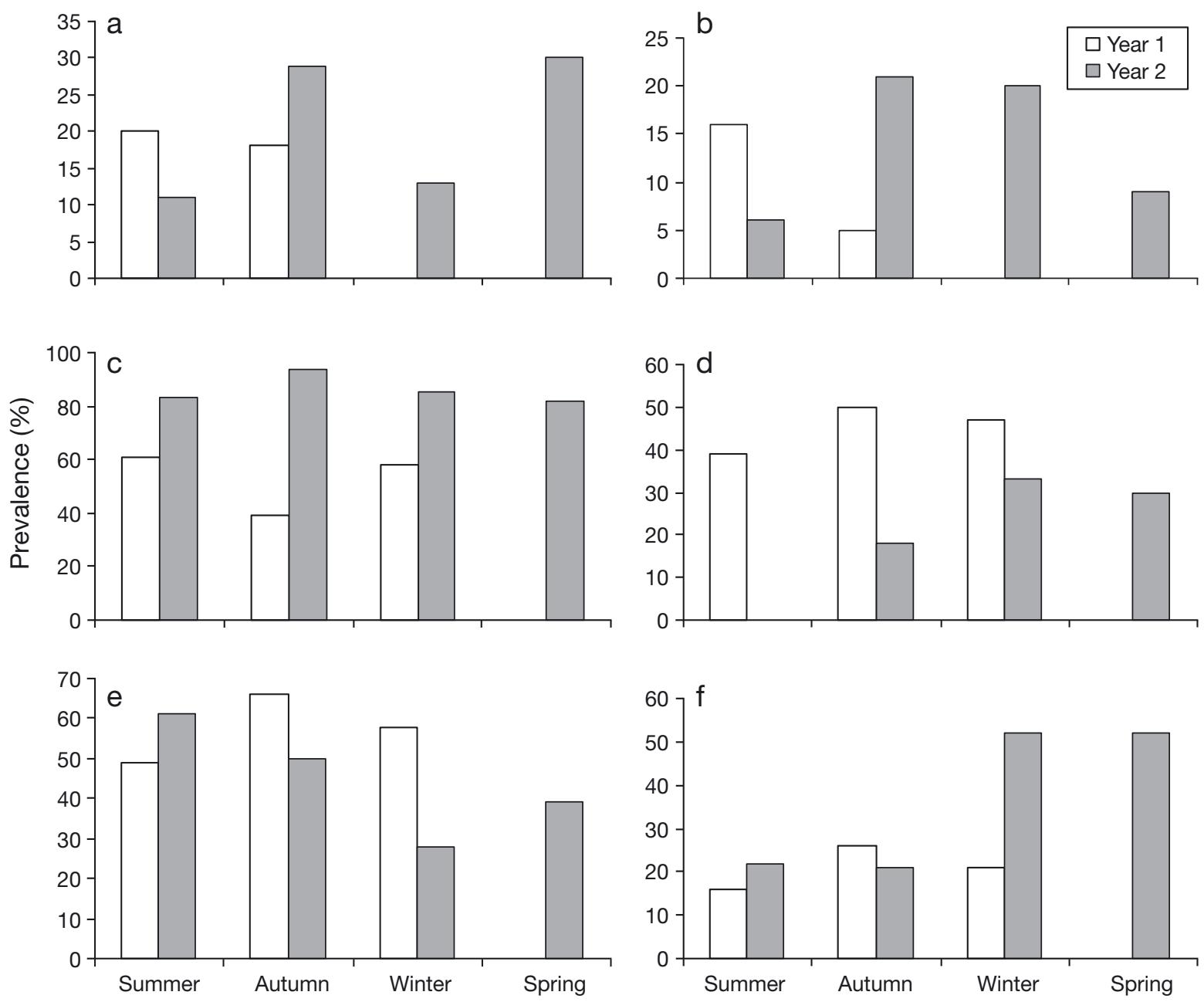

Fig. 3. Seasonal variation in helminth infection prevalence in Scinax nasicus. (a) Unknown echinostomatid species 1, (b) Catadiscus inopinatus, (c) Opisthogonimus sp. 2, (d) Travtrema aff. stenocotyle, (e) Bursotrema tetracotyloides, (f) Centrorhynchus sp. 
population, i.e. only a few host individuals are heavily infected, and that this distribution can have at least 2 main origins: (1) when infective stages are released in discrete waves, the parasites become highly aggregated in their hosts, and (2) through the exposure to intermediate hosts in which infective stages are aggregated. For digenean species, aggregation in Scinax nasicus is probably generated by the host movement, which tends to increase exposure to free-living contagious cercariae distributed within the host's habitat.

Also, the results show that in most cases there was no temporal variation in terms of species richness in the parasite communities; similarly, the abundance and prevalence of infections only showed large variation in a few helminth parasitic species, e.g. Travtrema aff. stenocotyle and Centrorhynchus sp. varied in prevalence among seasons, and Opisthogonimus sp. 2 and Travtrema aff. stenocotyle in abundance over time. These variations could be related to temporal constraints on the activity and foraging patterns of the snakes and birds which act as definitive hosts (see Table 3), thus affecting colonization and transmission of the parasite. Moreover, the low temporal variations observed in the other helminth infracommunities may be accounted for by the recruitment of immature parasites throughout different seasons of the year, coinciding with the prolonged reproductive activity of Scinax nasicus, which breeds during most of the year (see Hamann et. al. 2009), and combined with the persistent infection by adult parasites throughout the year in the definitive host. In this regard, temperature is not a significant factor in determining strong seasonal patterns in helminth parasitism in Corrientes, Argentina (Hamann 1999, 2006). Furthermore, the availability of invertebrate species such as insects, which may act as intermediate hosts, may also be constant during the seasons, and therefore the helminths' parameters are more stable over time.

Regarding host size, large-bodied frogs harbour more helminth parasite species than small-bodied frogs (see Hamann et al. 2006a). In the present study, the size of amphibians was a determining factor for parasite species richness, which suggests that larger frogs have been exposed to parasites for a longer time and also offer a larger surface area for parasitic attack, especially for infective larval stages. We also found that larger males were more parasitized (in terms of species richness) than larger females, suggesting that reduced immune function and behavioural differences between males and females may also explain the increasing levels of parasites (Folstad \& Karter 1992, Poulin 1996). Additionally, infrapopulations of Centrorhynchus sp. increase in abundance in larger hosts; this can occur as the result of accumulation of cystacanth larvae, or an increase in the numbers of a single prey type (e.g. coleopteran intermediate hosts) consumed. The host Scinax nasicus may be a good paratenic host for Centrorhynchus sp. (cystacanths) because it is eaten by birds, such as falconiformes (Smyth \& Smyth 1980).

Most authors agree that the helminth infracommunity structure in amphibians is depauperate and isolationist (Aho 1990, Muzzall 1991, Bolek \& Coggins 2003, Yoder \& Coggins 2007, Ibrahim 2008), while others found no fixed pattern, i.e. communities could be characterized variously along this continuum rather than as isolationist or interactive (Luque et al. 2005, Hamann et al. 2006a,b). In the present study, the helminth infracommunities of Scinax nasicus exhibited few abundant species, and poor species richness. In fact, we noted associations for those larval species whose individuals were located in different frog organs (Bursotrema tetracotyloides + unknown echinostomatid species 1), and in the same organs of the frog (Opisthogonimus sp. $2+$ Travtrema aff. stenocotyle). Likewise, we found a negative correlation between 2 species at different infection sites (e.g. Catadiscus inopinatus vs. B. tetracotyloides). There was no discernible pattern or structure to the infracommunities of $S$. nasicus, although these results provide evidence of their proximity to the isolationist extreme of the continuum, and they are depauperate when only the adult helminth species are considered. This might also indicate that there are numerous vacant niches in the frog digestive tract. On the other hand, the co-occurrence of metacercariae observed in this study could affect the structure of gastrointestinal helminths in the definitive hosts, such as snakes (see Poulin 2001).

Aho (1990) demonstrated that the habitat use and diet type (generalist vs. specialist) of the host can lead to associated differences in their parasite communities, and are important factors in the development of the latter. The present study also showed that the habitat preference plays an important role; in this sense, adult Scinax nasicus frogs live and feed almost exclusively in arboreal habitats, i.e. bromeliads, whereas they reproduce and develop into tadpoles in aquatic environments. Thus, the parasite fauna is dominated in both number of species and number of individuals by larval trematodes, particularly allogenic species whose definitive hosts are snakes, birds, and mammals, which can leave the immediate aquatic habitat thereby introducing the infective stage (Esch \& Fernandez 1994, Esch et al. 1997). By contrast, adult trematodes are poorly represented, which could be related to the frog's diet type that includes dipterans as the dominant item (Duré 1999). The digeneans found are characterized by having heteroxenous life cycles that take place mainly in aquatic (e.g. Catadiscus inopinatus, Glypthelmins sp., and Gorgoderina rochalimai) or ter- 
restrial (e.g. Mesocoelium monas; see Prudhoe \& Bray 1982) environments. Similarly, the less diverse nematological fauna and lower abundance of parasitic infections in $S$. nasicus reflects the limited contact with the terrestrial environment where the infective forms of the nematodes occur, e.g. Cosmocerca parva, and Cosmocerca podicipinus, which infect through skin penetration (Anderson 2000). This result contrasts with those found in leptodactylid frogs captured in the same area but characterized by different habitat use (semiaquatic and terrestrial); the latter show higher values for the ecological descriptors and indices of the nematode community (Hamann et al. 2006a,b). Additionally, the low infections of these nematodes found in S. nasicus agree with the previous reports of Bursey et al. (2001), and Goldberg et al. (2007) from other American Scinax species.

In conclusion, the community of helminths in Scinax nasicus suggests that the 'sit and wait' feeding strategy and the diet in particular of this frog were determining factors in the less diverse infections of adult helminths with complex life cycles. At the same time, the low vagility and the arboreal habitat preference of $S$. nasicus contributes significantly to the low occurrence of infection by skin-penetrating nematodes, while its trematode fauna shows high larval infections, resulting from acquisition of parasites following penetration by infective larvae (see Table 3), when the frogs visit the water to breed. Moreover, the helminth communities of $S$. nasicus are dominated by generalist metacercariae species that infect diverse amphibian hosts in the same area (Hamann et al. 2006a,b); frogs are susceptible to infection with cercariae in the tadpole stage (Hamann \& González 2009) and also after metamorphosis, which suggests that they are suitable second intermediate hosts for these metacercariae. In fact, these results may be alternatives to ensure transmission because $S$. nasicus occupies an intermediate position in the food web, being easy prey for potential definitive hosts, such as snakes, birds and mammals.

Acknowledgements. We are grateful to Dr. R. Poulin and Dr. M. Ostrowski de Núñez for helpful comments which greatly improved this manuscript. Financial support was provided by Consejo Nacional de Investigaciones Científicas y Técnicas (CONICET) of Argentina, through grant PIP 2945 to M.I.H.

\section{LITERATURE CITED}

Addinsoft (2004) Xlstat for excel, version 7.5. Addinsoft, New York, NY

Aho JM (1990) Helminth communities of amphibians and reptiles: comparative approaches to understanding patterns and processes. In: Esch GW, Bush AO, Aho JM (eds) Parasite communities: patterns and processes. Chapman \& Hall, London, p 157-196

Anderson RC (2000) Nematode parasites of vertebrates: their development and transmission, 2nd edn. CAB International, Oxford

Bell G, Burt A (1991) The comparative biology of parasite species diversity: internal helminths of freshwater fish. J Anim Ecol 60:1047-1063

Bolek MG, Coggins JR (2001) Seasonal occurrence and community structure of helminth parasites in green frogs, Rana clamitans melanota, from southeastern Wisconsin, U.S.A. Comp Parasitol 68:164-172

Bolek MG, Coggins JR (2003) Helminth community structure of sympatric eastern American toad, Bufo americanus americanus, northern leopard frog, Rana pipiens, and blue-spotted salamander, Ambystoma laterale, from southeastern Wisconsin. J Parasitol 89:673-680

Bolek MG, Snyder SD, Janovy J Jr (2009) Alternative life cycle strategies and colonization of young anurans by Gorgoderina attenuata in Nebraska. J Parasitol 95:604-616

Bursey CR, Goldberg SR, Parmelee JR (2001) Gastrointestinal helminths of 51 species of anurans from Reserva Cuzco Amazónico, Peru. Comp Parasitol 68:21-35

Bush AO, Lafferty KD, Lotz JM, Shostak AW (1997) Parasitology meets ecology on its own terms: Margolis et al. revisited. J Parasitol 83:575-583

Carnevali R (1994) Fitogeografía de la provincial de Corrientes. Ediciones Litocolor, Asunción

Cawthorn RJ, Anderson RC (1976) Development of Physaloptera maxillaries (Nematoda: Physalopteroidea) in skunk (Mephitis mephitis) and the role of paratenic and other hosts in its life cycle. Can J Zool 54:313-323

Combes C (1972) Influence of the behavior of amphibians on helminth life cycles. Zool J Linn Soc 51(Suppl 1):151-170

Crofton HD (1971) A quantitative approach to parasitism. Parasitology 62:179-193

Crump ML, Scott NJ Jr (1994) Visual encounters surveys. In: Heyer WR, Donnelly MA, McDiarmid RW, Hayek LC, Foster MS (eds) Measuring and monitoring biological diversity - standard methods for amphibians. Smithsonian Institution Press, Washington, DC, p 84-91

Dogiel VA (1964) General parasitology. Oliver \& Boyd, London

Duré MI (1999) Interrelaciones en los nichos tróficos de dos especies sintópicas de la familia Hylidae (Anura) en un área subtropical de Argentina. Cuad Herpetol 13:11-18

Elliot JM (1971) Some methods for the statistical analysis of samples of benthic invertebrates. Freshw Biol Assoc Sci Publ 25:1-156

Esch GW, Fernandez JC (1994) Snail-trematodes interactions and parasite community dynamics in aquatic systems: a review. Am Midl Nat 131:209-237

Esch GW, Bush AO, Aho JM (1990) Parasite communities: patterns and processes. Chapman \& Hall, London

Esch GW, Wetzel EJ, Zelmer DA, Schotthoefer AM (1997) Long-term changes in parasite population and community structure: a case history. Am Midl Nat 137:369-387

Esch GW, Barger MA, Fellis KJ (2002) The transmission of digenetic trematodes: style, elegance, complexity. Integr Comp Biol 42:304-312

Folstad I, Karter AJ (1992) Parasites,bright males, and the immunocompetence handicap. Am Nat 139:603-622

Fotedar DN, Tikoo R (1968) Studies on the life cycle of Cosmocerca kashmirensis Fotedar, 1959, a common oxyurid nematode parasite of Bufo viridis in Kashmir. Indian Sci Congr Assoc Proc 55:460

Frost D (2007) Amphibian species of the world: an online reference. Version 5.0 (Feb 2007) American Museum of Natural History, New York, NY. Available at http://research. amnh.org/herpetology/amphibia/index.php 
Gibson DI, Jones A, Bray RA (2002) Keys to the trematoda. CABI Publishing and The Natural History Museum, London

Gillilland MG, Muzzall PM (1999) Helminths infecting froglets of the northern leopard frog (Rana pipiens) from Foggy Bottom Marsh, Michigan. J Helminthol Soc Wash 66:72-77

Goater T, Goater CP (2001) Ecological monitoring and assessment network (EMAN) protocols for measuring biodiversity: parasites of amphibians and reptiles Canada. Available at http://eqb-dqe.cciw.ca/eman/ecotools/protocols/ terrestrial/herp_parasites/intro.html

Goldberg SR, Bursey CR, Caldwell JP, Vitt LJ, Costa GC (2007) Gatrointestinal helminths from six species of frogs and three species of lizards, sympatric in Pará State, Brazil. Comp Parasitol 74:327-342

Grabda-Kazubska B (1963) The life cycle of Metalepthophallus gracillimus (Lühe, 1909) and some observations on the biology and morphology of developmental stages of Letophalus nigrovenosus (Bellingham, 1844). Acta Parasitol Pol 11:349-370

> Gregory RD, Keymer AE, Harvey PH (1996) Helminth parasite richness among vertebrates. Biodivers Conserv 5: 985-997

Hamann MI (1999) Population biology of Spirocamallanus inopinatus (Travassos, Artigas et Pereira, 1928) (Nematoda, Camallanidae) in Serrasalmus spilopleura Kner, 1860 (Pisces, Characidae) from Corrientes, Argentina. Res Rev Parasitol 59:1-6

Hamann MI (2004) Seasonal maturation of Catadiscus propinquus (Digenea: Diplodiscidae) in Lysapsus limellus (Anura: Pseudisae) from an Argentinean subtropical permanent pond. Physis 59:29-36

Hamann MI (2006) Seasonal maturation of Glypthelmins vitellinophilum (Trematoda: Digenea) in Lysapsus limellus (Anura: Pseudidae) from an Argentinean subtropical permanent pond. Braz J Biol 66:85-93

> Hamann MI, González CE (2009) Larval digenetic trematodes of tadpoles of six amphibian species from Northeastern Argentina. J Parasitol 95:623-628

Hamann MI, Kehr AI, González CE (2006a) Species affinity and infracommunity ordination of helminths of Leptodactylus chaquensis (Anura: Leptodactylidae) in two contrasting environments from northeastern Argentina. J Parasitol 92:1171-1179

> Hamann MI, González CE, Kehr AI (2006b) Helminth community of Leptodactylus latinasus (Anura: Leptodactylidae) from Corrientes, Argentina. Acta Parasitol 51: 294-299

Hamann MI, Kehr AI, González CE, Duré MI, Schaefer EF (2009) Parasite and reproductive features of Scinax nasicus (Anura: Hylidae) from a South American subtropical area. Interciencia 34:214-218

Holm S (1979) A simple sequentially rejective multiple test procedure. Scand J Stat 6:65-70

Holmes JC, Price PW (1986) Communities of parasites. In: Anderson DJ, Kikkawa J (eds) Community ecology: pattern and process. Blackwell Scientific Publications, Oxford, p 187-213

Ibrahim MMI (2008) Helminth infracommunities of the maculated toad Amietophrynus regularis (Anura: Bufonidae) from Ismailia, Egypt. Dis Aquat Org 82:19-26

Jones MK (1987) A taxonomic revision of the Nematotaeniidae Lühe, 1910 (Cestoda: Cyclophyllidea). Syst Parasitol 10:165-245

Jones A, Bray RA, Gibson DI (2005) Keys to the trematoda. CABI Publishing and The Natural History Museum, London
Kehr AI, Manly BFJ, Hamann MI (2000) Influence of biotic and abiotic factors on helminth co-occurrences in Lysapsus limellus (Anura, Pseudidae) from an Argentinean subtropical area. Oecologia 125:549-558

King KC, Gendron AD, McLaughlin JD, Giroux I and others (2008) Short-term seasonal changes in parasite community structure in northern leopard froglets (Rana pipiens) inhabiting agricultural wetlands. J Parasitol 94:13-22

> Leigh WH (1946) Experimental studies on the life cycle of Glypthelmins quieta (Stafford, 1990), a trematode of frogs. Am Midl Nat 35:460-483

Luque JL, Martins AA, Tavares LER (2005) Community structure of metazoan parasites of the yellow Cururu toad Bufo ictericus (Anura, Bufonidae) from Rio de Janeiro, Brasil. Acta Parasitol 50:215-220

Magurran AE (2004) Measuring biological diversity. Blackwell Publishing, Oxford

Marcogliese DJ, King KC, Salo HM, Fournier M and others (2009) Combined effects of agricultural activity and parasites on biomarkers in the bullfrog, Rana catesbeiana. Aquat Toxicol 91:126-134

McAlpine DF (1997) Helminth communities in bullfrogs (Rana catesbeiana) green frogs (Rana clamitans), and leopard frogs (Rana pipiens) from New Brunswick, Canada. Can J Zool 75:1883-1890

McKenzie VJ (2007) Human land use and patterns of parasitism in tropical amphibian hosts. Biol Conserv 137: $102-116$

Moravec F (1977) Life history of the Rhabdochona phoxini Moravec, 1968 in the Rokytka Brook, Czechoslovakia. Folia Parasitol 24:97-105

Moravec F (1998) Nematodes of freshwater fishes of the Neotropical region. Academia Praha, Prague

Moravec F, Kaiser H (1994) Trichospirura amphibiophila n. sp. (Nematoda: Rhabdochonidae) in the frog Eleutherodactylus martinicensis from La Désirade, French Antilles. J Parasitol 80:121-125

Muzzall PM (1991) Helminth infracommunties of the frogs Rana catesbeiana and Rana clamitans from Turkey Marsh, Michigan. J Parasitol 77:366-371

> Muzzall PM, Gillillant MG, Summer CS, Mehne CJ (2001) Helminth communities of green frogs Rana clamitans Latreille, from southwestern Michigan. J Parasitol 87: 962-968

Ostrowski de Núñez M (1974) Sobre el ciclo biológico de Episthmium suspensum (Braun 1901) Travassos 1922. Rev Mus Argentino Cienc Nat Bernardino Rivadavia Inst Nac Investig Cienc Nat Zool 1:153-164

Ostrowski de Núñez M (1979a) Fauna de agua dulce de la república Argentina. IX. Sobre representantes de la fauna Paramphistomatidae (Trematoda). Physis 38:55-62

> Ostrowski de Núñez M (1979b) Ungewöhnliche Xiphidiocercarie aus Ampullaria canaliculata nebst Bemerkungen über Travtrema stenocotyle. Angew Parasitol 20:46-52

- Pearson JC (1960) On the life cycle of Neodiplostomum buteonis and the occurrence of a periprostate (Trematoda: Diplostomidae). J Parasitol 46:48

Pielou EC (1966) The measurement of diversity in different types of biological collections. J Theor Biol 13:131-144

Poulin R (1996) Sexual inequalities in helminth infections: a cost of being a male? Am Nat 147:287-295

> Poulin R (1997) Species richness of parasite assemblages: evolution and patterns. Annu Rev Ecol Syst 28:341-358

> Poulin R (2001) Interactions between species and the structure of helminth communities. Parasitology 122:S3-S11

> Poulin R (2005) Detection of interspecific competition in parasite communities. J Parasitol 91:1232-1235 
Price PW (1980) Evolutionary biology of parasites. Princeton University Press, Princeton, NJ

Prudhoe S, Bray RA (1982) Platyhelminth parasites of the amphibian. British Museum (Natural History), Oxford University Press, London

Schmidt GD (1985) Development and life cycles. In: Crompton DWT, Nickol BB (eds) Biology of the Acanthocephala. Cambridge University Press, Cambridge, p 273-305

Shannon CE, Weaver W (1949) The mathematical theory of communication. University of Illinois Press, Urbana, IL

Smyth JD, Smyth MM (1980) Frogs as host-parasite systems. I. An introduction to parasitology through the parasites of Rana temporaria, $R$. esculenta and $R$. pipiens. Macmillan Press, London

Southwood TR (1978) Ecological methods. Chapman \& Hall, London

Thomas JD (1965) The anatomy, life history and size allometry of Mesocoelium monodi Dollfus, 1929. J Zool 146:

Editorial responsibility: David Marcogliese, Montreal, Quebec, Canada
413-446

- Wetzel EJ, Esch G (1997) Infrapopulation dynamics of Halipegus occidualis and Halipegus eccentricus (Digenea: Heminuridae): temporal changes within individual hosts. J Parasitol 83:1019-1024

Yamaguti S (1961) Systema helminthum, Vol 3. The nematodes of vertebrates. Interscience New York, NY

Yamaguti S (1963) Systema helminthum, Vol 5. The Acanthocephala of vertebrates. Interscience, New York, NY

Yamaguti S (1975) A synoptical review of life histories of digenetic trematodes of vertebrates. Keigaku Publishing Company, Tokyo

Yoder HR, Coggins JR (2007) Helminth communities in five species of sympatric amphibians from three adjacent ephemeral ponds in southeastern Wisconsin. J Parasitol 93:755-760

Zar JH (1996) Biostatistical analysis, 3rd edn. Prentice-Hall, Upper Saddle River, NJ

Submitted: February 8, 2010; Accepted: August 2, 2010

Proofs received from author(s): November 22, 2010 